\title{
A BAND SELECTION METHOD FOR HIGH PRECISION REGISTRATION OF HYPERSPECTRAL IMAGE
}

\author{
Han Yang ${ }^{1}$, Xiaorun $\mathrm{Li}^{* 1}$ \\ College of Electrical Engineering, Zhejiang University, Hangzhou 310027, China - 1xrly@zju.edu.cn
}

KEY WORDS: Hyperspectral Image, High Spatial Resolution Image, Registration, CRLB Theory, Band Selection, Algorithm

\begin{abstract}
:
During the registration of hyperspectral images and high spatial resolution images, too much bands in a hyperspectral image make it difficult to select bands with good registration performance. Terrible bands are possible to reduce matching speed and accuracy. To solve this problem, an algorithm based on Cram'er-Rao lower bound theory is proposed to select good matching bands in this paper. The algorithm applies the Cram'er-Rao lower bound theory to the study of registration accuracy, and selects good matching bands by CRLB parameters. Experiments show that the algorithm in this paper can choose good matching bands and provide better data for the registration of hyperspectral image and high spatial resolution image.
\end{abstract}

\section{INTRODUCTION}

Hyperspectral images are wildly applied in agriculture, forestry, geosciences, and astronomy as hyperspectral images have a wealth of spectral information that can detect nondetectable substances in panchromatic and multispectral spatial resolution images (Yuan Zhou, 2017). Compared with hyperspectral images, multispectral images have higher spatial resolution, so collaborative processing of hyperspectral images and high spatial resolution images in the same area will help to effectively detect and recognize unknown targets in hyperspectral images. Due to different sensor resolution, different optical paths, or different image mechanisms, the phenomena of translation, rotation and scaling may occur between images. Precise registration of multisource remote sensing image is the co-processing of hyperspectral image and high spatial resolution.

Grayscale and texture differences between different bands in hyperspectral images and high spatial resolution images are an important reason for poor matching accuracy. So choosing appropriate bands of hyperspectral image to match is the key to improve the registration accuracy of hyperspectral image and high spatial resolution image. The method of PCA was derived to reduce hyperspectral data dimension in (YU Xian-chuan, 2013), (Lei Wang, 2007). A new band will be generated based on all bands of hyperspectral images through the method of PCA. Although the method accounts for minimizing the loss of important information for the later stages of the methodology, this new band will lose some details of the original image, resulting in increased registration difficulty. In addition, Hang Chen et al (CHEN Hang, 2013) selected a band of hyperspectral images according to the sharpness of the texture information to participate in the registration. However, this method isn't based on the quantitative evaluation index of registration accuracy, and the selected band registration accuracy needs to be improved.

In (Bond, 2005), CRLB theory was applied to the field of registration, and the lower limit of noise variance of image was measured by the lower CRLB to evaluate the image registration performance. More recently, (Yetik I S, 2006) applied CRLB theory to the evaluation of registration parameters, and theoretically deduced the method of calculating the lower bound of CRLB for different registration methods. In (Xu Baoshu, 2011) and (LI Jing, 2009), the lower limit of CRLB for registration parameters was calculated in different scenarios. It was proved experimentally that the lower limit of CRLB could be used as an effective tool for image registration performance evaluation. In this paper, a high-precision registration band selection method based on CRLB theory is proposed, which not only reduces the computational complexity of the data but also selects the band with good matching performance in hyperspectral image.

\section{RELATED WORK}

\subsection{Affine Deformation Model}

Affine deformation model (Gong M, 2014) is the most common geometric deformation model. when using an affine model for registration, the geometric relationship between the pending map and the baseline is as follow:

$$
\left[\begin{array}{c}
s_{i x} \\
s_{i y}
\end{array}\right]=a^{*}\left[\begin{array}{l}
\cos \theta,-\sin \theta \\
\sin \theta, \cos \theta
\end{array}\right] *\left[\begin{array}{l}
o_{i x} \\
o_{i y}
\end{array}\right]+\left[\begin{array}{l}
t_{i x} \\
t_{i y}
\end{array}\right]
$$

Points in the reference image $S=\left[s_{1}, s_{2}, \ldots, s_{l}\right]$, the number of which is L, among them $s_{i}=\left[s_{i x}, s_{i y}\right]^{T}$. Points in the sensed image $\mathrm{O}=\left[o_{1}, o_{2}, \ldots, o_{l}\right]$, the number of which is $\mathrm{L}$, among them $o_{i}=\left[o_{i x}, O_{i y}\right]^{T}$, a is the scale factor, and $\theta$ is the rotation angle. $\left(t_{i x}, t_{i y}\right)$ is the amount of translation in the $\mathrm{x}, \mathrm{y}$ direction. It is supposed that $\mathrm{D}$ is the deformation matrix, $\mathrm{T}$ is the translation vector, the above equation can be abbreviated as:

$$
s_{i}=D^{*} o_{i}+T
$$

The real position in the sensed image corresponded to the point set $\mathrm{S}$ of the reference image is $R=\left[r_{1}, r_{2}, \ldots, r_{l}\right]$, among 
them $r_{i}=\left[r_{i x}, r_{i y}\right]^{T}$. It is supposed that there is only Gaussian white noise between the corresponding points and real points, then $o_{i}=r_{i}+n_{o i} \quad$, covariance matrix is $\Sigma_{o}=\left(\operatorname{diag}\left[\sigma_{o x}^{2}, \sigma_{o y}^{2}\right]\right), \quad \sum_{s}=\left(\operatorname{diag}\left[\sigma_{s x}^{2}, \sigma_{s y}^{2}\right]\right)$, among then $n_{\text {oi }}=\left[n_{\text {oix }}, n_{\text {oiy }}\right]^{T}, \quad n_{s i}=\left[n_{\text {six }}, n_{\text {siy }}\right]^{T}$, so:

$$
\left[\begin{array}{c}
s_{i x} \\
s_{i y}
\end{array}\right]=a^{*}\left[\begin{array}{l}
\cos \theta,-\sin \theta \\
\sin \theta, \cos \theta
\end{array}\right] *\left[\begin{array}{l}
r_{i x} \\
r_{i y}
\end{array}\right]+\left[\begin{array}{l}
t_{i x} \\
t_{i y}
\end{array}\right]+\left[\begin{array}{l}
n_{s i x} \\
n_{s i y}
\end{array}\right]
$$

The corresponding abbreviation is:

$$
s_{i}=D^{*} r_{i}+T+n_{s i}=h_{i}+n_{s i}
$$

\subsection{CRLB Theory}

The lower limit of CRLB is proposed for the problem of parameter estimation, and the lower bound is set for the variance of unbiased estimator. The unbiased estimator variance of parameters can only approach the lower CRLB limit without reaching. (Steven M. Key, 1993)

Lemma 1: It is assumed that the probability density function $\mathrm{p}(\mathrm{x} ; \theta)$ of a parameter estimate satisfies the "regularity" condition $E\left[\frac{\partial \ln p(x ; \theta)}{\partial \theta}\right]=0$ for all $\theta$, when the expectation is taken with respect to $\mathrm{p}(\mathrm{x} ; \theta)$. Then the variance of any unbiased estimator $\hat{\theta}$ must satisfy

$$
\operatorname{var}(\hat{\theta}) \geq \frac{1}{-\frac{\partial^{2} \ln p(x[0] ; \theta)}{\partial \theta^{2}}}
$$

$\frac{1}{-\frac{\partial^{2} \ln p(x[0] ; \theta)}{\partial \theta^{2}}}$ is the minimum variance defined as the lower CRLB for parameter $\theta$.

Lemma 2: It is assumed that the probability density function $\mathrm{p}(\mathrm{x} ; \theta)$ of several parameter estimates satisfies the "regularity" conditions $E\left[\frac{\partial \ln p(\boldsymbol{x} ; \boldsymbol{\theta})}{\partial \theta}\right]=\boldsymbol{0}$ for all $\boldsymbol{\theta}$, when the expectation is taken with respect to $\mathrm{p}(\mathrm{x} ; \theta)$. Then the covariance matrix of any unbiased estimator $\hat{\theta}$ satisfies

$$
\boldsymbol{C}_{\hat{\theta}}-\boldsymbol{I}^{-1}(\boldsymbol{\theta}) \geq \boldsymbol{0}
$$

Among them $[\boldsymbol{I}(\boldsymbol{\theta})]_{i j}=-E\left[\frac{\partial^{2} \ln p(\boldsymbol{x} ; \boldsymbol{\theta})}{\partial \boldsymbol{\theta}_{i} \partial \boldsymbol{\theta}_{j}}\right]$ is defined as FIM information matrix. Covariance lower bound matrix is $\operatorname{var}\left(\hat{\theta}_{i}\right)=\left[\boldsymbol{C}_{\hat{\theta}}\right]_{i i} \geq\left[\boldsymbol{I}^{-1}(\boldsymbol{\theta})\right]_{i i}$

\section{METHODOLOGY}

\subsection{Calculation Method of CRLB Lower Limit}

Since the variance of the parameter $\theta$ can reflect the accuracy of the parameter estimation, the smaller the variance, the more accurate the estimation. Therefore, it is feasible to use the CRLB lower bound to evaluate the parameters.

We can see from the second section of chapter two, the lower CRLB is solved by evaluating the likelihood function of the parameter estimates by the second-order derivative. Therefore, when applying the CRLB theory to the evaluation of registration parameters, a likelihood function should be constructed first, and then the likelihood function is derived. The following is the derivation process.

The registration model uses the affine deformation model, as shown in equation (3) and (4). The deformation parameters are $A=\left[a, \theta, t_{x}, t_{y}\right]^{T}, H=\left[h_{1}, h_{2}, \ldots, h_{L}\right], C_{o}, C_{s}$ are diagonal matrix of $\Sigma_{0,} \Sigma_{g}$. The log likelihood function of two image deformation parameters is:

$$
\ln p(\boldsymbol{O}, \boldsymbol{S}: \boldsymbol{A})=-\frac{1}{2} *\left[(\boldsymbol{O}-\boldsymbol{R})^{T} C_{0}^{-1}(\boldsymbol{O}-\boldsymbol{R})+(\boldsymbol{S}-\boldsymbol{G})^{T} C_{*}^{-1}(\boldsymbol{S}-\boldsymbol{G})\right]+\text { const }
$$

Therefor the various components of the Fish information matrix are:

$$
\begin{gathered}
J_{A_{m}, A_{n}}=-E\left[\frac{\partial^{2} \ln (p(O, S: A))}{\partial A_{m} A_{n}}\right] \\
J_{A_{m}, A_{n}}=\frac{1}{\sigma_{s x}^{2}} \sum_{i=1}^{L}\left(\frac{\partial h_{i x}}{\partial A_{m}} * \frac{\partial h_{i x}}{\partial A_{n}}\right)+\frac{1}{\sigma_{s y}^{2}} \sum_{i=1}^{L}\left(\frac{\partial h_{i y}}{\partial A_{m}} * \frac{\partial h_{i y}}{\partial A_{n}}\right)
\end{gathered}
$$

$$
\operatorname{CRLB}(\boldsymbol{A})=J^{-1}(\boldsymbol{A})
$$

\subsection{Registration Performance Evaluation}

Compared with the lower limit of CRLB of the registration parameters, the registration position difference can more intuitively reflect the image registration performance. So, on the basis of the lower limit of the registration parameter, the lower limit of the position accuracy of the registration is calculated, and the registration performance is more intuitively reflected. The following is simple derivation of the calculation of position accuracy. Position error can be written as:

$$
\begin{gathered}
\varepsilon=\frac{1}{L} \sum_{i=1}^{L} S_{i}-\frac{1}{L} \sum_{i=1}^{L}\left(D r_{i}+T\right)=\varepsilon_{1}-\varepsilon_{2} \\
\operatorname{Cov}\left(\varepsilon_{1}\right)=\operatorname{diag}\left(\left[\frac{\sigma_{s x}^{2}}{L}, \frac{\sigma_{s y}^{2}}{L}\right]\right)
\end{gathered}
$$


It is assumed $\quad g(A)=\varepsilon_{2} \quad, \quad$ among them $A=\left[a, \theta, t_{x}, t_{y}\right]^{T}$, combine equation [9], then we can get the following equation:

$$
\operatorname{Cov}\left(\varepsilon_{2}\right) \geq \frac{\partial g(A)}{\partial A} J^{-1}(A)\left[\frac{\partial g(A)}{\partial A}\right]^{T}
$$

$\operatorname{Cov}(\varepsilon) \geq \frac{\partial g(A)}{\partial A} J^{-1}(A)\left[\frac{\partial g(A)}{\partial A}\right]^{T}+\operatorname{diag}\left(\left[\frac{\sigma_{s x}^{2}}{L}, \frac{\sigma_{s y}^{2}}{L}\right]\right)$

The diagonal elements of the matrix obtained are the lower limits of the position error in the $\mathrm{x}$ and $\mathrm{y}$ directions.

\subsection{Algorithm Flow}

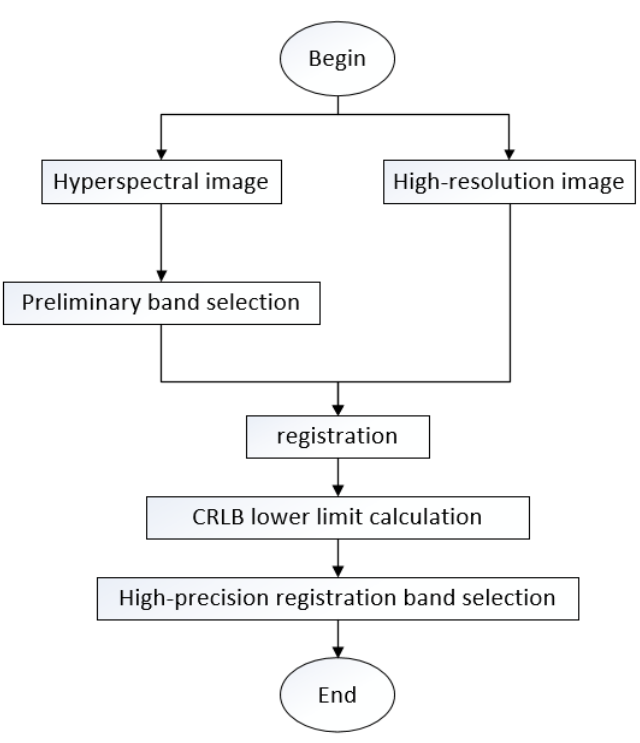

Figure1: high-precision registration band selection algorithm flow chart

The purpose of matching band selection algorithm is to select suitable band in a large number of bands of hyperspectral images for registration, so as to reduce the amount of matching computation and provide a band set with better matching performance for subsequent registration. Algorithm flow chart is shown in Figure one. The process of the algorithm is divided into the following sections. Firstly, a preliminary band selection is made for the hyperspectral image to select some bands with rich information and little relevance. Then the preliminary selected band and high-resolution images are registered, meanwhile record the registration results. Finally, the CRLB lower limit of registration parameters of different bands is calculated according to the registration result. Several bands with good registration performance are selected according to the CRLB lower limit.
3.3.1 Preliminary Band Selection: The high data dimensionality of hyperspectral images increases the burden on data computation, storage, and transmission(Siwei,2017). As there are too many bands in the hyperspectral image, the matching band selection algorithm first makes a preliminary band selection of the hyperspectral image to reduce the amount of data in order to reduce the subsequent calculation. Among lots of band selection method, KL divergence method takes full account of the correlation between the bands, and the selected band through KL divergence is representative, so we use the method of KL divergence to preliminarily select the bands.

The concrete method is as follows: firstly, the grayscale distribution of each band of the hyperspectral image is counted, and then KL divergence value of each band against it's next one-third band is calculated. The specific calculation method see equation 15 .

$$
\begin{aligned}
& D(P \| Q)=\sum(P(x) \log (P(x) / Q(x)))+ \\
& \sum(\mathrm{Q}(x) \log (Q(x) / P(x)))
\end{aligned}
$$

3.3.2 Registration: In order to pick the best matching band set, a preliminary matching process is necessary. After a preliminary match, we can pick out some of the better matching bands through analyzing the matching parameters. The main purpose of this process is to calculate the registration parameters, rather than getting accurate registration result. Therefore, registration methods such as region-based registration, feature-based registration are to meet the requirements. Hyperspectral image has to many bands, so wo choose SIFT, a common point feature based algorithm (Lowe D G, 2004), accounting for the algorithm speed.

In this process, feature points are extracted and described by SIFT algorithm, and then the Euclidean distance between feature points of reference image and sense image is calculated and compared. Affine deformation model is used to calculate the parameters of registration deformation.

3.3.3 high-precision matching band selection: After preliminary match, we get a series of matching results. The above describes how to calculate the CRLB lower limit through the registration parameters. This step applies the above formula to calculate the CRLB lower limit and the lower limit of position accuracy for each set of registration parameters. According to the location accuracy of the lower limit of the data, we can choose the band with smaller registration error, that is high-precision matching band.

\section{EXPRIMENT}

The main purpose of this section is to prove that the band selected by the method proposed in this paper is a band that matches well. Therefore, the experiment selected hyperspectral image bans according to the high-precision registration bands selection algorithm. The selected bands registration results and PCA registration results were compared to observe the selected bands' registration performance.

\subsection{Simulated Image Data Set}

The reference image in a 2005 aerial hyperspectral image with a total of 124 bands, 10 meters resolution (size: $325 * 500$ ). The 
sensed image is selected from the reference image, delarges 4 times and rotate 20 degrees.

\subsection{Evaluation Criteria}

The root mean square error(RMSE) is used as an index to evaluate the registration accuracy. $\llbracket x_{i}, y_{i} \rrbracket$ are randomly generated, and the corresponding point pairs $\left[x_{i}^{b}, y_{i}^{t} \rrbracket\right.$ are obtained by the affine transformation model formed by the required parameters, the corresponding point pairs

$\llbracket x_{i}^{t s}, y_{i}^{t 5} \rrbracket$ are obtained by the affine transformation model formed by the real parameters. The formula for calculating the root mean square error is as follows:

$$
R M S E=\sqrt{\frac{1}{N} \sum_{i=1}^{N}\left(\left(x_{i}^{\prime \prime}-x_{i}^{\prime}\right)^{2}+\left(y_{i}^{\prime \prime}-y_{i}^{\prime}\right)^{2}\right)}
$$

\subsection{Result and Analysis}

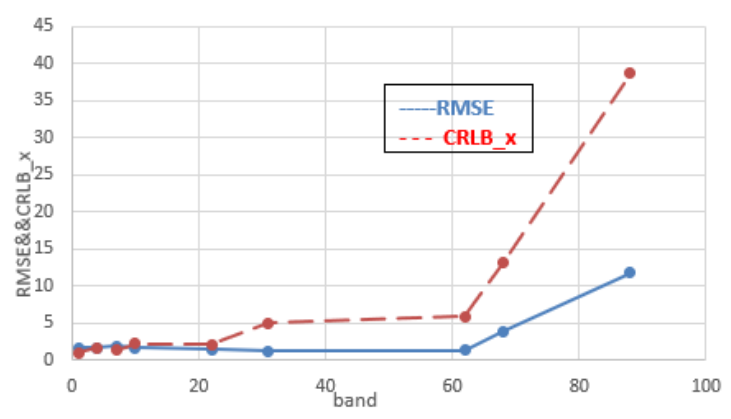

Figure 2 Trend chart of RMSE and CRLB_x

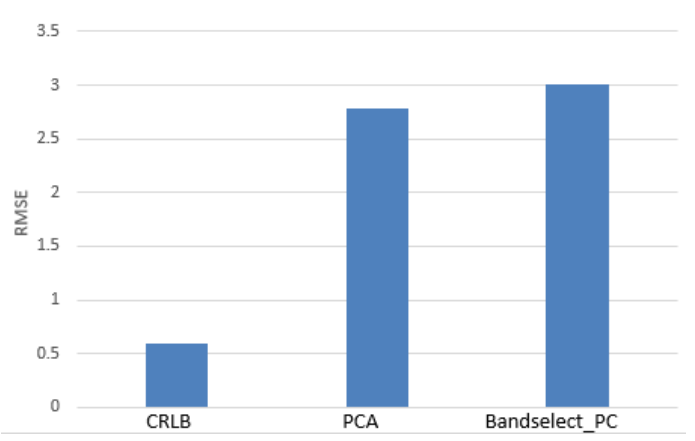

Figure 3 RMSE of three methods

Comparing the curve of the CRLB lower limit and the root mean square in figure 2.The $\mathrm{x}$ axis of figure 2 is the number of hyperspectral bands. It can be found that the trend of these two indexes are consistent, which shows that in general the CRLB lower limit can measure registration accuracy instead of the root mean square. From figure 3, it is clear that the registration result of the band selected by the CRLB lower limit is more accurate than the band generated by PCA or PCA after normal band selection.

\section{CONCLUSION}

In this paper, an algorithm based on CRLB theory for selecting hyperspectral high-precision matching bands is proposed. Main steps have three: preliminary band selection, registration, CRLB lower limit calculation. The experiment shows that the bands selected by the method in this paper is a band set with better registration performance.

The method can make more accurate judgment of the registration result without knowing the true deformation parameters between the reference image and the sensed image, then selects suitable registered bands. Based on the work, more accurate registration is possible between hyperspectral image and high-resolution image.

\section{ACKNOWLEDGEMENTS (OPTIONAL)}

This work was supported by the National Natural Science. Foun dation of China (no.61671408) and the Joint Funds of the Ministry of Education of China (no.6141A02022314).

\section{REFERENCES}

Pham T., Schutte K, J., 2005, Performance of optimal registration estimators, Proc Spie.

Chen H, Du X., Xia L., Cheng X., J., 2013, registration method for hyperspectral image based on control points, Journal of Equipment Institute, 24(3):109-113.

Gong M, Zhao S, Liu J, J., 2014, A Novel Coarse-to-Fine Scheme for Automatic Image Registration Based on SIFT and Mutual Information, IEEE Transactions on Geoscience \& Remote Sensing, 52(7):4328-4338.

Wang L, Liu X., D., 2007, study of image registration technique and application, xi'an university of electronic science and technology.

Li J, Huang P., Wang X., Pan X., J., 2009, registration precision study based on crlb theory for optical base image and sar image.

Lowe D., J., Distinctive Image Features from Scale-Invariant Key points, International Journal of Computer Vision, 60(2):91110.

Feng S., Yuki I, Mario P, Marco, J., 2017, hyperspectral band selection from statistical wavelet models, IEEE transactions on geoscience \& remote sensing, 55(4):2111-2123

Steven M., M., 1993, Fundamentals of Statistical Signal Processing Estimation Theory, page27-33.

Xu B., Shi Z., Chen F., J., 2011, characterization of image registration error based on cramer-rao lower bound, Journal of instrumentation.

Yetik I., Nehorai A, J., 2006, Performance bounds on image registration, IEEE Transactions on Signal Processing, 54(5):1737-1749.

Yu X., Lu Z., Hu D., J., 2013, review of remote sensing image registration technique, Optical precision engineering, 21(11):2960-2972

Zhou Y, Anand R, Paul D, C., 2017, nonrigid registration of hyperspectral and color images with vastly different spatial and spectral resolutions for spectral unmixing and pansharpening, 
The International Archives of the Photogrammetry, Remote Sensing and Spatial Information Sciences, Volume XLII-3, 2018 ISPRS TC III Mid-term Symposium "Developments, Technologies and Applications in Remote Sensing", 7-10 May, Beijing, China

IEEE Conference on Computer Vision and Pattern Recognition Workshops, 